\title{
A new control strategy for shape memory alloys actuators
}

Pierre-Antoine Gédouin ${ }^{1, a}$, Cédric Join ${ }^{2,3}$, Emmanuel Delaleau ${ }^{1}$, Jean-Matthieu Bourgeot ${ }^{1}$, Shabnam Arbab Chirani ${ }^{1,4}$, and Sylvain Calloch ${ }^{4}$

1 Université Européenne de Bretagne, École Nationale d'Ingénieurs de Brest, Laboratoire Brestois de Mécanique et des Systèmes - EA 4325, Technopôle Brest-Iroise, CS 73 862, 29238 Brest, France.

2 Centre de recherche en automatique de Nancy, UMR CNRS 7039, Université Henry Poincaré-Nancy I, Faculté des sciences et techniques, BP 239, 54506 Vandœuvre, France.

3 Projet ALIEN - INRIA Futurs.

4 Université Européenne de Bretagne, École Nationale Supérieure d'Ingénieurs, Laboratoire Brestois de Mécanique et des Systèmes - EA 4325, 2 rue François Verny, 29806 Brest, France.

\begin{abstract}
Shape memory alloys (SMA) are more and more integrated in engineering applications. These materials with their shape memory effect permit to simplify mechanism and to reduce the size of actuators. SMA parts can easily be activated by Joule effect but their modelling and consequently their control remains difficult, it is principally due to their hysteretic thermomechanical behaviour. Another difficulty is that the characteristics of the material are timevarying, especially during cyclic loadings. So, most of successful control strategy applied to SMA actuator are particularly heavy and used the Preisach model or neural networks to model the hysteretic behaviour of these material but this kind of models are difficult to identify and to use in real time. That is why this study deals with an application of the new framework of model-free control and restricted model control applied to a SMA spring based actuator. This control strategy is based on new results on fast derivative s estimation of noisy signals, its main advantages are: its simplicity, its robustness and the fact that it is easy to compute. Experimental results and comparisons with PID control are exposed that demonstrate the efficiency of this new control strategy despite thermal perturbations.
\end{abstract}

\section{Introduction}

Shape memory alloys (SMA) offer the possibility to recover a known shape after a thermomechanical cycle. This property, known as the "shape memory effect", is due to the transition between the two crystallographic phases in their composition. This variation of shape, controlled by temperature variation, may be used in the development of actuators (see e.g., [1] and [2]). SMA can easily be heated by Joule effect, but their control is not completely solved and it is principally due to their hysteretic behaviour (see [3]). Another difficulty is that the characteristics of the material are time-varying, especially during cyclic loadings. Phases kinetic transformations and 3-dimensional models are proposed in [4], [5], [6] and [7]. These complex models can render very subtle properties of SMA, but often need to compute a finite element code, what is not suitable for real time control. On the opposite side, Robotics research have been done on SMA actuator by using simpler model and classical control method. A lot of control strategies have been applied to SMA actuators, classical PID loop are used in [8], [9]

\footnotetext{
${ }^{a}$ e-mail: gedouin@enib.fr
} 
and [10]. A feedforward path is added in [11]; the feedforward command is obtain by using a Preisach model for the hysteretic effect. In [12] feedforward scheme is also used but the hysteretic behaviour is described by a Duhem differential hysteresis model. In $[13,14]$ a feedforward loop is combined with sliding mode control to obtain robustness, the feedforward path is respectively given by neural network and by a physical model. An $H_{\infty}$ loop based on physics modelling is design in [15]. Passivity property of the system is used in [16] to prove stability of a proposed proportional law. Nonlinear control techniques based on the Lie algebra are also used in [17]. Even if their model are quite good enough, using a dynamic model for computing the control law, need firstly to identify the model parameter. As we have already mention the model parameter of SMA vary during cycling, then a classical model based control is ineffective or particularly complex. We report our experience, where industrial partners still explain that in order to realise the process control, the part of process modelling represents $90 \%$ of project global time. Indeed, to define the physical model structure, to identify unknowns parameters, to collect experimental data, to valid the model are never simple. However, how is it possible to control a complex process without any model?

In this paper, a solution to this difficult problem is proposed. The proposition is based on some new results in the framework of "Model-free control" (see [18-21]). The approach uses a derivative estimation (see [22-24]) which provides good results even if signals are corrupted by noise. Thus a non-physical model valid a very short part of time is estimated and permits classical control design.

The present work constitutes an extension of a previous paper [25], in which only simulations have already highlighted the advantages of the proposed method in the area of SMA actuators.

The paper is organised as follows: The next Section is a short review and introduction to the new "Model-free control" and explains the design of a control law within this new framework; Sec. 3 develops the model-free control of a shape memory alloys actuator and gives experimental results. Sec. 4 concludes the paper and raises some perspectives.

\section{Model free control}

Model free control is a very recent approach to nonlinear control that has been introduced in [26], (See [18] for a thorough presentation). A first industrial and convincing application is reported in $[21]$.

\subsection{Derivatives of noisy signals}

Firstly, we recall basic of derivative estimation. Interested reader might refer to [22] for a complete presentation. We consider a signal $y$ that is available through a measurement $y_{m}$ corrupted by some additive noise $\varpi$, i.e. $y_{m}=y+\varpi$. The objective is to estimate time derivatives of signal $y(t)$, up to a finite order, from its measurement $y_{m}$.

The Taylor expansion of $y$ around 0 reads:

$$
y(\tau)=\sum_{n=0}^{\infty} y^{(n)}(0) \frac{\tau^{n}}{n !}
$$

Approximate $y(t)$ in the interval $[0, T], T>0$, by the polynomial $y_{N}(\tau)=\sum_{n=0}^{N} y^{(n)}(0) \frac{\tau^{n}}{n !}$ of degree $N$. The operational ${ }^{1}$ analogue (see [27]) $Y_{N}(s)$ of $y_{N}(\tau)$ is given by:

$$
Y_{N}(s)=\frac{y(0)}{s}+\frac{\dot{y}(0)}{s^{2}}+\cdots+\frac{y^{(N)}(0)}{s^{N+1}}
$$

\footnotetext{
1 Reader not familiar with operational calculus can just think in terms of Laplace transform to understand the development of the derivatives estimators.
} 
It is possible to isolate each coefficient $y^{(i)}(0)$ appearing in the previous expression by applying a convenient operator to $Y_{N}(s)$ (see [22] for details ${ }^{2}$ ). Indeed:

$$
\forall i=0, \ldots, N, \quad \frac{y^{(i)}(0)}{s^{2 N+1}}=\frac{(-1)^{i}}{N !(N-i) !} \cdot \frac{1}{s^{N+1}} \cdot \frac{d^{i}}{d s^{i}} \cdot \frac{1}{s} \cdot \frac{d^{N-i}}{d s^{N-i}}\left(s^{N+1} Y_{N}(s)\right)
$$

Having in mind that the operator $\frac{1}{s^{\alpha}}$ corresponds to the function $t \mapsto \frac{t^{\alpha-1}}{(\alpha-1) !}$, that the operator $\frac{d}{d s}$ corresponds to the multiplication in the time domain by $-t$ and using the Cauchy formulae to transform a multiple integral in a simple one:

$$
\int_{0}^{T} \int_{0}^{\tau_{\alpha-1}} \cdots \int_{0}^{\tau_{1}} f(\mu) d \mu d \tau_{1} \ldots d \tau_{\alpha-1}=\int_{0}^{T} \frac{(T-\mu)^{\alpha-1}}{(\alpha-1) !} f(\mu) d \mu
$$

One obtain in the time domain the expression of $y^{(i)}(0)$ as:

$$
y^{(i)}(0)=\int_{0}^{T} P(\mu ; T) y_{N}(\mu) \mathrm{d} \mu
$$

where $P(\mu ; T)$ is polynomial in $\mu$ and $T$. Notice that (3) gives the calculation of $y^{(i)}(0)$ from an integral on the time interval $[0, T]$ for a given small $T>0$. As $\left.\frac{d^{i} y(t-\mu)}{d \mu^{i}}\right|_{\mu=0}=(-1)^{i} y^{(i)}(t)$ it is possible to express $y^{(i)}(t)$ as an integral which evolves values of $y_{N}$ on the time interval $[t-T, t]$ :

$$
y^{(i)}(t)=(-1)^{i} \int_{0}^{T} P(\mu ; T) y_{N}(t-\mu) d \mu
$$

A simple estimator of the derivative $y^{(i)}(t)$ is then obtained from the noisy signal $y_{m}$ by:

$$
\left[y^{(i)}(t)\right]_{e}=(-1)^{i} \int_{0}^{T} P(\mu ; T) y_{m}(t-\mu) d \mu
$$

which is deduced from (3) by replacing $y_{N}$ by $y_{m}$ in (4). Note that the integral operation plays the role of low-pass filter and reduced the noise that corrupts $y_{m}$ The choice of $T$ results in a trade-off: the larger is $T$, the smaller is the effect of the noise ( the larger is $T$ the better is integrals low pass filtering) and the larger is the error due to truncation.

In practise, (5) is evaluated at each sample time $t=k \cdot T_{s}, k=0,1, \ldots$ as a discrete sum using basic trapezoidal method or Simpson method to calculate integrals:

$$
\left[y^{(i)}\left(k T_{s}\right)\right]_{e}=\sum_{i=0}^{n_{s}} w(i) P\left(i T_{s} ; n_{s} T_{s}\right) y_{m}\left((k-i) T_{s}\right)
$$

with $n_{s}$ the number of samples used in the time window and $n_{s} T_{s}=T$.

\subsection{Model-free control design}

Assume we have a plant whose we do not know any model. For the sake of simplicity of the presentation we assume that this plant is single-input and single-output. The control input is denoted by $u$ and the output is denoted as $y$. As seen in the previous section, we are able to estimate on-line some derivatives of $y$ and $u$. Model-free control consist in trying to estimate via the input and the output measurements what can be compensated by control in order to

\footnotetext{
${ }^{2}$ Note that those operators are not unique, we have chosen here to use the ones with the least order of integration for the sake of simplicity of the presentation.
} 
achieve a good output trajectory tracking. This implies the construction of a purely numerical model of the plant that can be written as:

$$
y^{(\nu)}=F+\alpha u
$$

where $\alpha \in \mathbb{R}$ is a non-physical constant ${ }^{3}$ design parameter; $F \in \mathbb{R}$ represents all what is unknown on the system and can be compensated from the knowledge of the input-output behaviour of the system; the order $\nu \in \mathbb{N}$ of the numerical model (7) is also a design parameter that can be arbitrarily chosen.

Eqn. (7) should not be confused with a "black-box" identified model. In the present approach, (7) is update at each sampling time from the knowledge of the input-output behaviour of the unmodeled plant. At sampling time $k$ (i.e. $t=k T_{s}$, where $T_{s}$ denotes the sampling period), the estimation of $F$ reads:

$$
[F(k)]_{e}=\left[y^{(\nu)}(k)\right]_{e}-\alpha u(k-1)
$$

where $\left[y^{(\nu)}(k)\right]_{e}$ is the estimation of the $\nu$-st derivative of the output that can be laid at time $k$ and $u(k-1)$ is the control input that has be applied to the plant during the previous sampling period.

Based on the numerical knowledge of $F$ the control for sampling period $k$ is calculated on $(7)$ as a simple cancellation of the non-linear terms $F$ plus a closed loop tracking of a reference trajectory $t \mapsto y^{*}(t)$ :

$$
u(k)=\underbrace{-\frac{[F(k)]_{e}}{\alpha}}_{\text {NL Cancellation }} \underbrace{+\frac{\left[y^{*(\nu)}(k)\right]_{e}+\Delta(\epsilon(k))}{\alpha}}_{\text {Closed loop tracking }}
$$

where $\epsilon(k)=y(k)-y^{*}(k)$ is the tracking error and $\Delta(\epsilon(k))$ is a closed-loop feedback controller base on the tracking error. Note that the term $-\frac{[F(k)]_{e}}{\alpha}+\frac{\left[y^{*(\nu)}(k)\right]_{e}}{\alpha}$ is also the "nominal control" in the "flatness-based" control of (7) (see e.g., [28-30]). When the closed loop controller is of "PID" type, Model free control can be named as "intelligent PID" ( $i$-PID) (see [18]).

This control scheme is summarised in Fig. 1

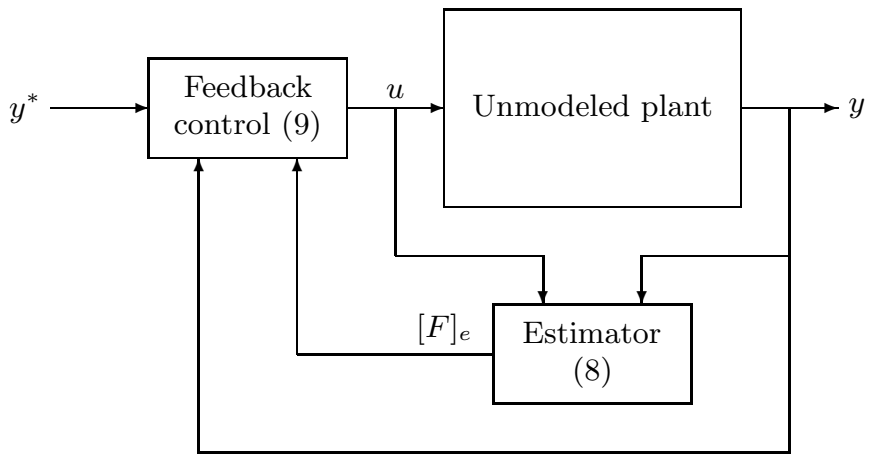

Fig. 1. Model free control

\footnotetext{
${ }^{3}$ Note that this parameter could also be chosen time varying for some applications.
} 


\section{Prototype of SMA Actuator}

A simple prototype of SMA spring actuator has been built, a picture of the actuator is given in Fig. 2. The upper extremity of a Ni-Ti spring is electrically isolated and fixed to rigid structure and the lower extremity is attached to a $450 \mathrm{~g}$ mass. The mechanism of this kind of actuator is decomposed into three steps. Firstly, when the temperature is constant and lower than the austenite start temperature $A_{s}$, the SMA spring is purely martensitic and the tensile force provided by the mass deforms the SMA spring to an initial deflection. Secondly the spring is heated, when its temperature goes past $A_{s}$, the martensite begins to change into austenite, so the spring tries to achieve its high temperature shape consequently the mass goes up. Finally during cooling austenite changes into martensite so the force provided by the mass permit the mass to go down.

For this actuator, the spring is heated by joule effect and cooled by free convection. The electric current crossing the spring is measured using a shunt resistance and a voltage amplifier, we also measure electric voltage with a voltage amplifier and the position of the mass using a laser sensor. We use an input output board connected to a computer with RTAI/Linux coupled with an electric power generator to acquire and generate real time control signals.

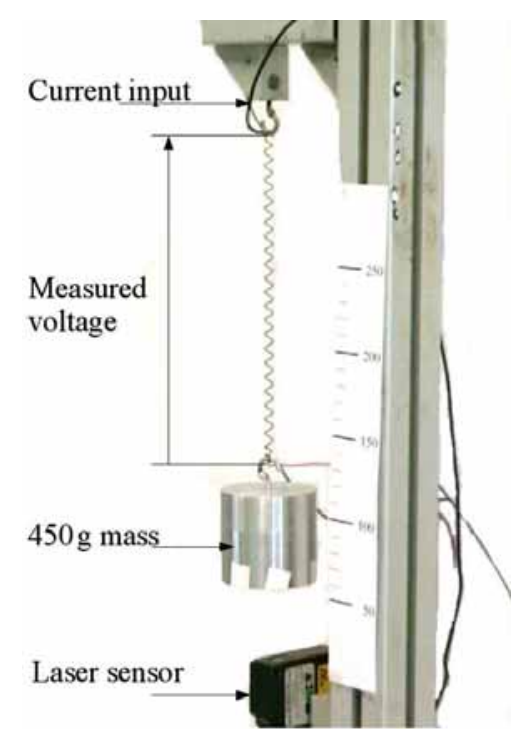

Fig. 2. Prototype of a SMA spring based actuator.

\subsection{Position control development}

For this application the very simple first-order local numerical model

$$
\dot{y}=F+\alpha u
$$

is considered. The control input $u$ corresponds the electric power crossing the SMA spring and the output $y$ is the measured vertical position of the mass. According to section 2.2, the control is given by:

$$
u=\frac{1}{\alpha}\left(-[F]_{e}+\dot{y}^{\star}+K_{p}\left(y-y^{\star}\right)\right)
$$

where $K_{p}$ is a positive constant. Note that as (10) is here first order, a simple proportional controller is enough to ensure convergence of the error to zero. This controller is called an $i$-P controller. 


\subsection{Experimental results}

The previous control law was applied to our SMA actuator. The performances in closed loop are compared with the performances obtained with a PI controller ${ }^{4}$ for two scenarios.

\section{Scenario 1 (Fig. 3 ):}

We present classical closed loop step response with thermal disturbance. In this scenario the mass has to reach a reference position of $70 \mathrm{~mm}$. when the actuator is control with $i$-P controller, we can observe that the mass reaches the reference position in approximately 8 seconds without overshoot whereas a $17 \mathrm{~mm}$ overshoot and $34 \mathrm{~s}$ time response are observed when the actuator is control by a PI controller. This overshoot should be reduced (reducing the integral gain) nevertheless rejecting perturbations would be bad. At time $t=56 \mathrm{~s}$ a thermal perturbations is applied using a fan. We can observe that the perturbation is rejected faster by the $i$-P controller than the PI one.

Scenario 2 (Figs. 4 and 5 ):

We present classical tracking. We can observe that tracking is very good when the actuator is controlled by an $i$-P (see Fig 3) whereas it is unsatisfactory for the PI (see Fig 4). A A first solution would be to increase the gains of the PI controller but for this experiments the choice of gains level results in a trade-off because the larger are the gains the better are performances in transient however high level gains degrade performances in steady-state.

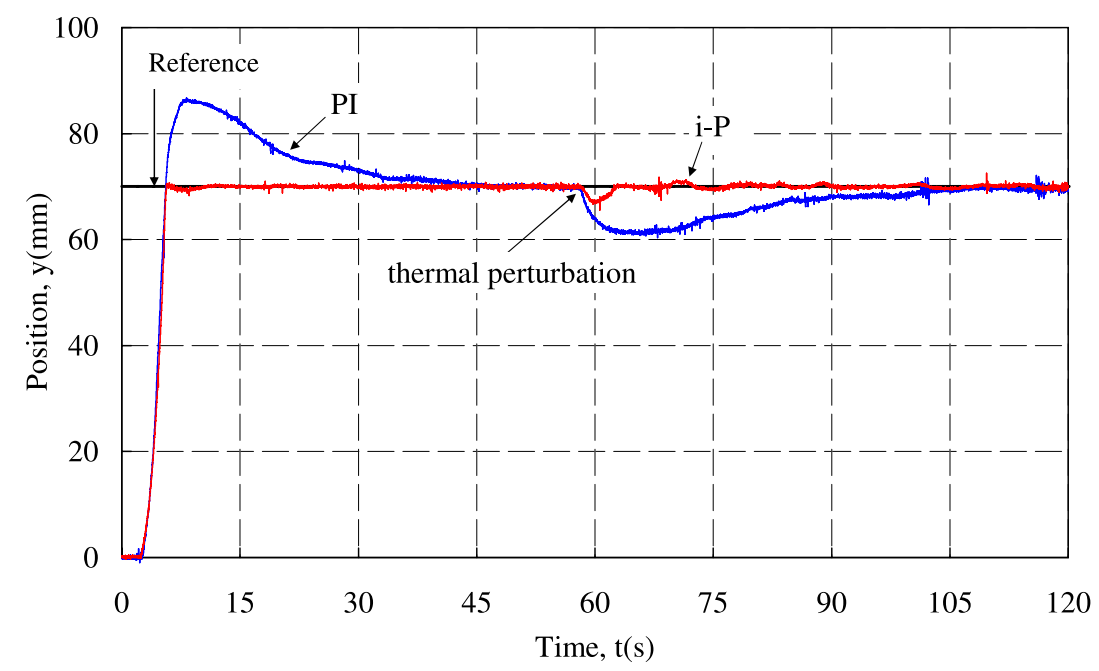

Fig. 3. Step response of the actuator with thermal disturbance (PI and $i$-P)

\section{Conclusion}

This paper presents a convincing application of the new model-free control in the area of SMA actuators control, a field in which control-model is especially difficult to obtain. Experimental results on a SMA spring based actuators show that the robustness towards thermal perturbations and tracking performances of this controller are better than the PI ones, whereas the design of the two controller needs the same numbers of tuning parameters. Finally, we are very

\footnotetext{
${ }^{4}$ The Classical control involves an integral terms in order to avoid static error.
} 


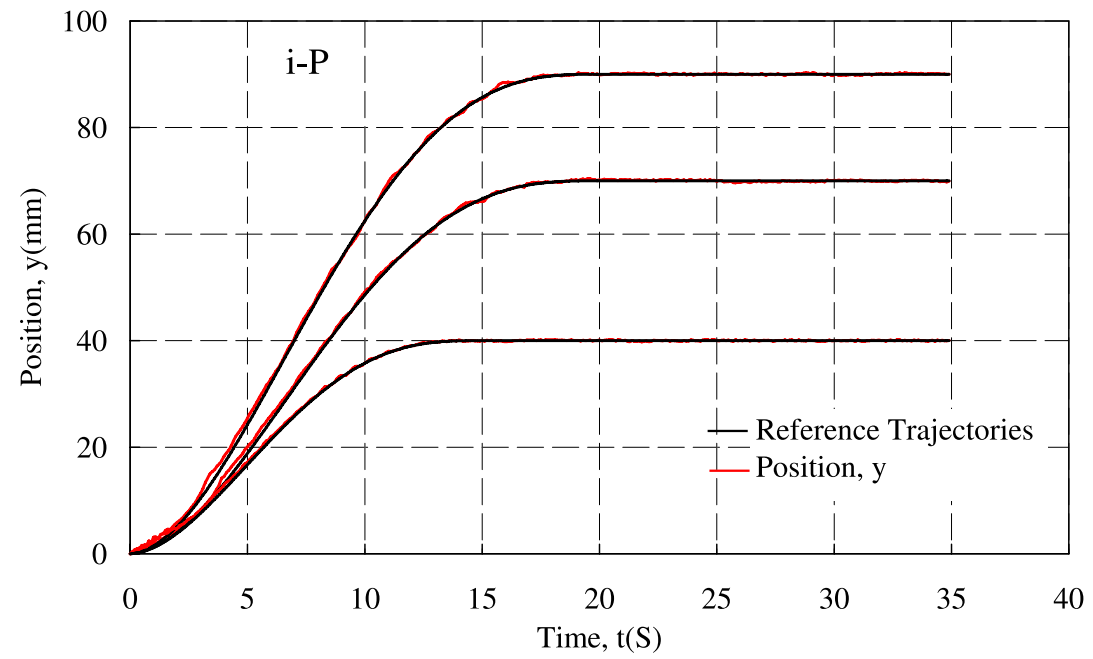

Fig. 4. Classical tracking when the actuator is controlled with an $i$-P controller.

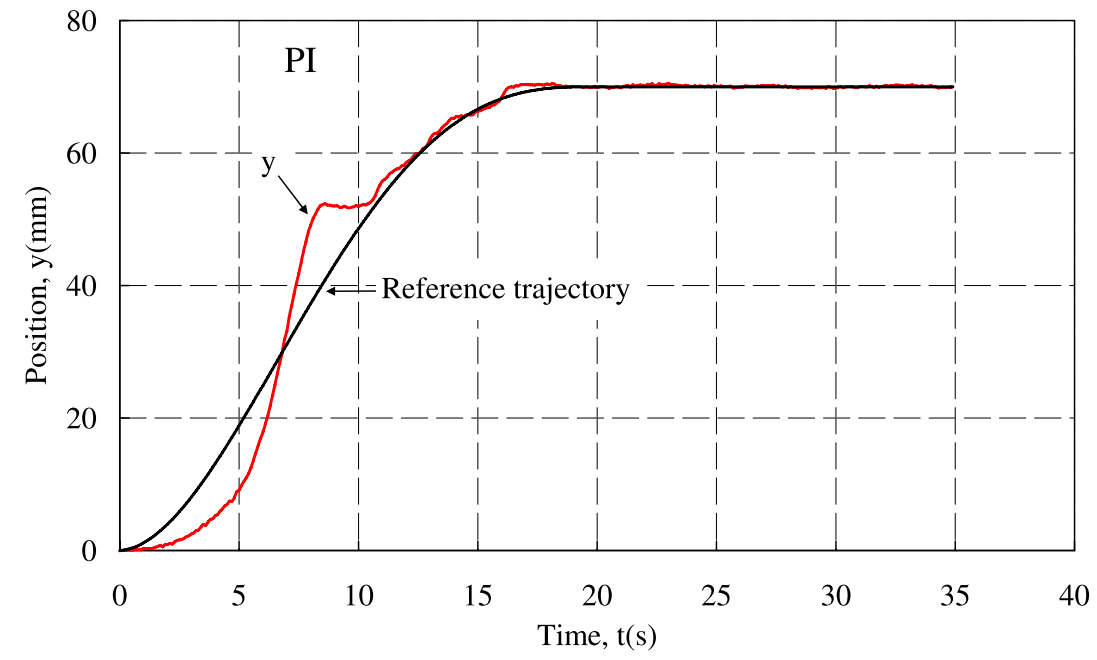

Fig. 5. Classical tracking when the actuator is controlled with a PI controller.

confident to be able to efficiently control SMA micro actuators as micro servomotors in a very short time.

\section{Acknowledgment}

This work was supported in part by the Agence nationale de la recherche (France) - Project "MAFESMA", in part by Brest métropôle océane, in part by Conseil génénéral du Finistère. P.-A. Gédouin is partially supported by Brest métropôle océane.

\section{References}

1. J. Peirs, D. Reynaerts, H. Van Brussel. In Proceedings of The International conference on new actuator, 77-80 (Bremen, Germany, 2002)

2. M. Kohl, B. Krevet, E. Just. Sens. Actuators A Phys 98646 (2002)

3. E. Patoor, A. Eberhardt, M. Berveiller. Mechanics of phase transformation and shape memory alloys 18923 (1994) 
4. S. Leclerc, C. Lexcellent. I. Mech. Phys. Solids 44, 6953 (1996)

5. P. Sittner, D. Vokoun, G. N. Dayananda, et al. Materials Science and Engineering A286 298 (2000)

6. S. Arbab Chirani, D. Aleong, C. Dumont, et al. Journal de Physique IV 112205 (2003)

7. C. Bouvet, S. Calloch, C. Lexcellent. European Journal of Mechanics A/Solids 2337 (2004)

8. M. Calin, A. Bertsch, N. Chaillet, et al. In Proceedings of the 1997 IEEE/RSJ Intelligent Robots and Systems, 33-34 (Grenoble, France, 1997)

9. E. Shameli, A. Alasty, H. Salaarieh. Mechatronics 15471 (2005)

10. E. P. Da Silva. Materials and design 281592 (2007)

11. S. Majima, K. Kodama, T. Hasegawa. IEEE Trans. Control Systems Technology 954 (2001)

12. S. M. Dutta, F. H. Ghorbel, J. B. Dabney. In International Symposium on Intelligent Control, 1007-1012 (Limassol, Cyprus, 2005)

13. G. Song, V. Chaudhry, C. Batur. Smart materials and structures 12223 (2003)

14. R. Romano, E. Aoun Tannuri. Mechatronics (2009). Available online 26 April 2009

15. J. Jayender, R. Patel, S. Nikumb, et al. In Proceedings of the 44th IEEE Conference on Decision and Control, and the European Control Conference 2005 (Seville, Spain, 2005)

16. D. R. Madill, D. Wang. IEEE Trans. Control Systems Technology 6, 4473 (1998)

17. H. Benzaoui, N. Chaillet, C. Lexcellent, et al. Smart Structures and Materials 1999: Mathematics and Control in Smart Structures 3667337 (1999)

18. M. Fliess, C. Join. In 15th IFAC Symposium on System Identification (SYSID 2009) (IFAC, SaintMalo France, 2009). http://hal.inria.fr/inria-00372325/en/

19. M. Fliess, C. Join, H. Sira-Ramirez. In Proc. 14th IFAC Symposium on System Identification (SYSID'06) (Newcastle, Australia, 2006). (In French), http://hal.inria.fr/inria-00000824/fr/

20. M. Fliess, C. Join, M. Mboup, et al. In Proc. Conférence internationale francophone d'automatique (CIFA'06) (2006). (In French), http://hal.inria.fr/inria-00001139/fr/

21. C. Join, J. Masse, M. Fliess. In Proc. $2^{e}$ Journées Identification et Modélisation Expérimentale (JIME'06) (Poitiers , France, 2006). (In French), http://hal.inria.fr/inria-00096695/fr/

22. M. Mboup, C. Join, M. Fliess. Numerical Algorithms 50, $4 \quad 439$ (2008). http://hal.inria.fr/inria-00319240/fr/

23. M. Mboup, C. Join, M. Fliess. In 15th Mediterrean Conference on Control and Automation (MED'07) (Athenes, Greece, 2007). http://hal.inria.fr/inria-00142588/fr/

24. M. Fliess, C. Join, H. Sira-Ramírez. International Journal of Modelling, Identification and Control (IJMIC) 4, 112 (2008). http://hal.inria.fr/inria-00158855/fr/

25. P.-A. Gédouin, C. Join, E. Delaleau, et al. In 17th IFAC World Congress (Seoul, Corée du sud, 2008). http://hal.inria.fr/inria-00261891/en/

26. M. Fliess, C. Join, M. Mboup, et al. In Confrence internationale francophone d'automatique (CIFA 2006) (IEEE, Bordeaux, France, 2006)

27. J. Mikusiński. Operational calculus. 2nd edn. (PWN \& Oxford University Press, 1983)

28. M. Fliess, J. Lévine, P. Martin, et al. Internat. J. Control 61, 61327 (1995)

29. V. Hagenmeyer, E. Delaleau. Internat. J. Control 76537 (2003)

30. V. Hagenmeyer, E. Delaleau. Automatica J. IFAC 391941 (2003) 